\title{
Performance Comparison of Near-Field Focused and Conventional Phased Antenna Arrays at $140 \mathrm{GHz}$
}

\author{
Dinesh Acharya, Joonas Kokkoniemi, Aarno Pärssinen, and Markus Berg \\ Centre for Wireless Communications (CWC), University of Oulu, P.O. Box 4500, 90014 Oulu, Finland \\ Email: \{forename.surname\}@oulu.fi
}

\begin{abstract}
The future high frequency systems require very high antenna gains to cope with the large channel losses. In order to obtain large gain and flexible beamforming, large antenna arrays are often considered. With hundreds or thousands of antenna elements, transmit energy is highly concentrated on the target. However, large numbers of antenna elements make the antennas very large compared with the wavelength. Therefore, it is likely for the user to be in the near field of the array. In this region, the maximum antenna gain is obtained by focusing the energy on the desired locations. The downside is a need to know the exact locations of the radios. This paper focuses on analysis of the traditional linear phase beam steering and near field focusing beam steering and the impact of the user location uncertainty on the achievable antenna gain. The uncertainty in the user location can arise, for instance, due to user mobility. The results show that the near field focusing gives superior gain in the near field of the antenna array, but is more sensitive to the user location information than the linear phase beam steering.

Index Terms-D band communications, millimeter wave communications, beamforming, near-field communications
\end{abstract}

\section{INTRODUCTION}

The future wireless communication systems are seeking the peak data rates from the millimeter wave (mmWave, $30-300 \mathrm{GHz})$ and terahertz $(\mathrm{THz}, 300 \mathrm{GHz}-10 \mathrm{THz})$ frequencies. These frequencies give opportunity to utilize very large bandwidths that allow very high theoretical data rates. Especially beyond fifth generation (B5G) communication systems are expected to benefit from the mmWave and $\mathrm{THz}$ bands. While being highly heterogeneous to satisfy application specific needs, the extreme data rates on B5G systems are expected to be achieved at $+100 \mathrm{GHz}$ sub- $\mathrm{THz}$ frequencies [1].

The high frequencies bring high path losses. This causes a need for high gain antennas at both ends of the communication link to reduce the overall loss. High gain antennas, on the other hand, bring need for beamforming in a desired direction to establish the communication links. In order to obtain high antenna gains and agile beamforming, antenna arrays are often considered [2], [3]. To achieve tens of decibels of antenna gain, very large numbers of antenna elements are required, a hundred or thousands of those. This is due to the maximum possible antenna gain of an antenna array is equal to the number of the antenna elements when the individual antenna responses perfectly and constructively sum at the wanted location or direction. A large antenna array tends to have large dimensions compared with wavelength. This pushes the nearfield (NF) of the antenna array far away from it. Therefore, it is likely that in many future applications the communications happens in the NF of the antennas [4].

Traditionally, phased arrays rely on linear phase shift beam steering (LPBS) [5]-[7]. This gives a straightforward way to maximize the antenna gain in the far-field (FF) of the array. In the NF, the maximum gain is obtained with near field focusing (NFF) [8]. In this beam steering method, the antenna phases are calculated from the distances of the individual elements to the target location in order to achieve constructive summation of the electric fields and the maximum gain. However, this requires not only the beam steering direction, but also the distances from each antenna element to desired location.the exact path lengths.

In order to establish communications link, the channel is estimated first to calculate the optimal beamformers. However, there can be inaccuracy in the estimate, e.g., due to user movement decreasing the estimate over time. This requires constant updating of the channel estimates to adjust the beam steering directions. In this paper, NFF and LPBS are analysed in the NF of the antenna array. The main focus of this paper is to analyse the impact of the user position uncertainty on the beamforming gain of extremely large antenna arrays in the $\mathrm{NF}$ at $140 \mathrm{GHz}$ frequency. Above $100 \mathrm{GHz}$ frequencies very high antenna gains lead to very large antenna arrays, and higher probability of user being in the NF. By utilizing NFF, significant gain can be achieved over LPBS, but the NFF is also far more sensitive to the inaccuracy of the exact user position, as it will be shown in the numerical results and illustrated in Fig. 1.

There are several papers on the NFF for various applications [6], [9]-[11]. For instance, authors in [12] provided an idea for the wireless power transfer (WPT) in the radiative NF, high resolution imaging using array NFF was shown in [10], radio frequency identification with NFF has been considered, e.g., in [6], NFF behavior of intelligent reflecting surfaces (IRS) has been studied in [9], non-contact sensing was presented in [13] and many more. Furthermore, [11] presents focused beam array implementation for a planar array with two focal distances at $10 \mathrm{GHz}$ central frequency. A detailed analysis of channel measurement as well as analysis for number of user positions is done in [14]. As such, the NFF has been shown to be very promising for NF applications. Herein, we append to the existing works by analysing the NFF with imperfect user position to analyse its performance in realistic wireless communication scenarios. To the best of our knowledge, there 


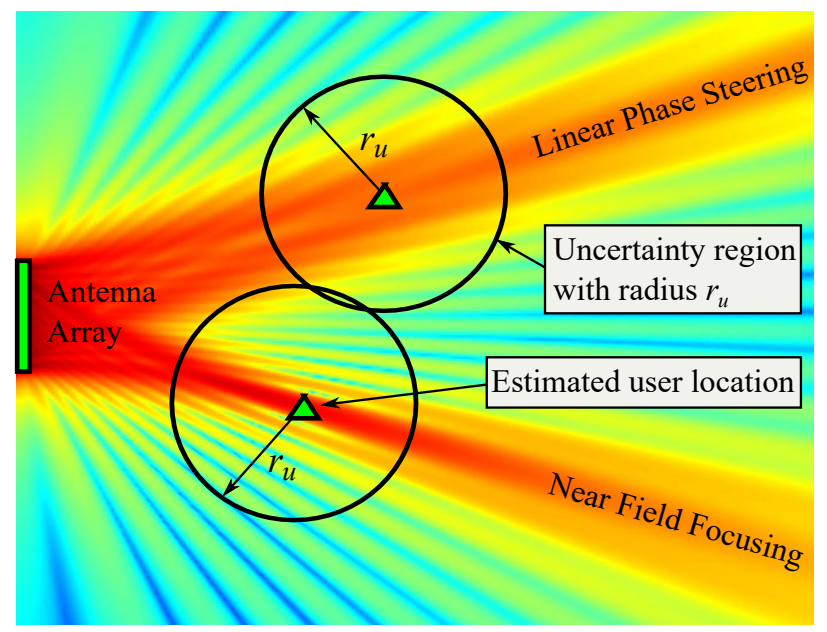

Fig. 1. The system model considered in this paper showing the estimated user locations, uncertainty regions, and antenna patterns.

are no other works on this topic.

The rest of this paper is organized as follows. Section II illustrates the system model and the problem formulation, the system geometry and the antenna models and signal models, Section III gives the numerical results, and finally Section IV concludes the paper.

\section{SySTEM MODEL}

This section gives the system model and its geometry, and the mathematical models for the considered beam steering methods and the user position uncertainty, as well as the signal model.

\section{A. General System Model}

The general system model considered herein is given in Fig. 1. Furthermore, the detailed system geometry with LPBS and NFF is given in Fig. 2. An electrically large antenna array is composed of large numbers of small antenna elements. The array can be considered as a single antenna structure when studying the signal propagation. Antenna array designing plays a vital role in the system performance, as the resulting signal strength depends on superimposed waves propagated from individual antenna elements. In LPBS, the beam is steered at a desired steering angle by calculating linear phase shift between the antenna elements, whereas in NFF, the idea is to control the phase of antenna array in such a way that the electric field sum constructively at the focus point. Plane wave and spherical wave concept arises from the distance between antenna array and user location. When the size of antenna array is very large in comparison with the wavelength, it is likely for a user to be in the NF of the array. Two approaches, namely NFF and LPBS are analyzed by generalizing propagation to two-dimensional (2D) geometry. Thus, we assume linear array in this study instead of planar array. It allows to study physically very large antennas. In the future work, we will extend the analysis to three-dimensional (3D) space, although, the fundamental operation principle is the same as in 2D space.

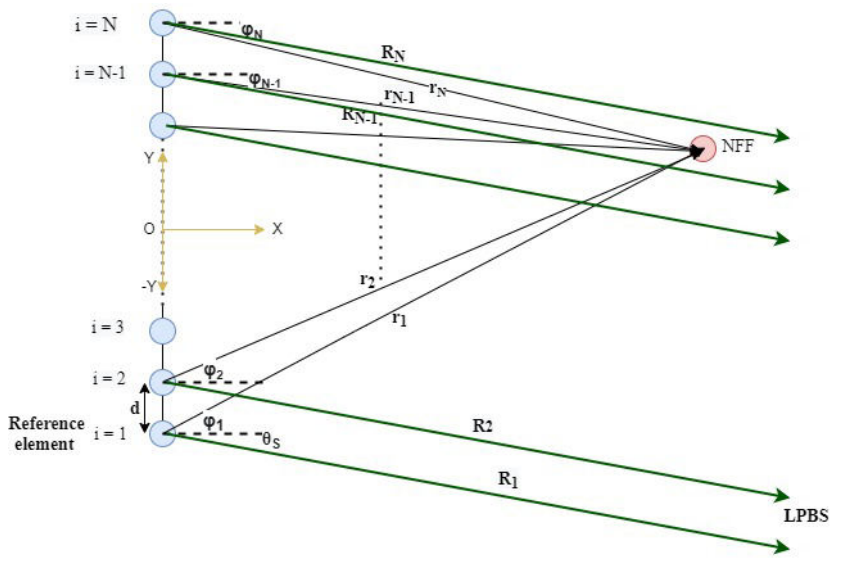

Fig. 2. System geometry for NFF and LPBS with $N$ element linear antenna array.

However, the path loss model is assumed to be 3D by applying spherical expansion of the waves in the space, but the antennas and user drop spaces are 2D in this work.

The main contribution of this paper is the analysis of the impact of the user position accuracy on the beamforming gain for LPBS and NFF. The NFF gives the maximum gain in the NF due to perfect alignment of the incoming signal phases. This requires knowledge of the user position, i.e., one extra degree of freedom compared to the LPBS which only requires the direction. The both methods give the same gain in the FF. However, and as it can be intuitively deduced based on Figs. 1 and 2, the NFF is far more sensitive to the exact user position, where imperfect position information impairs the achievable gain. This will be shown in the numerical results with implications it gives. In Fig. 1 two circle represents uncertainty region with radius $r_{u}$.

\section{B. Radiation Field Calculation}

The wave is propagating in $2 \mathrm{D}$ plane based on the steering angle or focus point as shown in Fig. 2. The NF and FF regions of antenna array can be given as [2], [5]

$$
F_{n}<\frac{2 D^{2}}{\lambda} \leq F_{f}
$$

where $F_{n}$ and $F_{f}$ are the NF and FF, respectively, $D$ is the maximum dimension of antenna, and $\lambda$ is the wavelength of the electromagnetic (EM) wave. In close proximity of the antenna, the electric field changes rapidly with spatial position due to spherical signal propagation.

A linear array with $N$ identical antenna element is placed on $\mathrm{y}$-axis and centered at the origin of coordinate system as shown in Fig. 2. The size of linear array antenna is $D=(N-1) d$, where $d$ is the antenna element spacing. The phases of the antenna elements are given in the next two section for the LPBS and NFF.

1) Linear Phase Beam Steering: The LPBS is a conventional beam steering approach which is based on steering angle $\left(\theta_{s}\right)$ obained by element-wise linear phase shifts [7]. This 
phase shift $(\Delta)$ between two successive elements is calculated as

$$
\Delta=\frac{2 \pi}{\lambda} d \sin \left(\theta_{s}\right) \text {. }
$$

2) Near Field Focusing: The NFF produces a focused beam at focus point. The exact phase of an antenna element depends on its distance to the focus point and the EM wavelength, as the NFF requires aligning the phases at the focus point. The overall electric field at any given point in space is a summation of signal contributions from individual antenna elements. Thus, the phase required to focus spherical wave at defined focus point can be computed as [6], [11]

$$
\phi_{i}=\frac{2 \pi}{\lambda} r_{i}
$$

where $r_{i}$ is the distance between the focus point and the antenna element, and it can be expressed as

$$
r_{i}=\sqrt{\left(y-x_{i}\right)^{2}+\left(x-y_{i}\right)^{2}},
$$

where $x$ and $y$ represent the position of focus point. $x_{i}$ and $y_{i}$ gives the position of the $i$ th antenna element.

3) Array Factor: The array factor $A_{f}$ gives the radiation pattern of the antenna at some observation point. It is based on geometry and the excitation phase of antenna array [2], [5], [8]. For a uniform linear array with $N$ element, $A_{f}$ can be expressed as,

$$
A_{f}=\frac{1}{\sqrt{N}} \sum_{i=1}^{N} a_{i} e^{-j \psi_{i}},
$$

where, $a_{i}$ weight factor, $\psi_{i}=(i-1) \Delta$ for LPBS, and $\psi_{i}=$ $2 \pi d_{i} / \lambda-\phi_{i}$ for NFF, where $d_{i}$ is the vector length from the $i$ th antenna element to the spatial coordinates of the observation point.

\section{Signal Model}

We consider free space path loss (FSPL) channel model in line-of-sight path between the antenna array and user location. Path loss is given as $P_{L}(r)=(4 \pi r)^{2} / \lambda^{2}$ [3], where, $r$ is a distance between the transmitter and the receiver. Utilizing the above array factor, the total received power $P_{R x}$ in general case can be calculated as

$$
P_{R X}=\frac{P_{T x} G_{R X}}{N}\left|\sum_{i=1}^{N} \frac{a_{i}}{\sqrt{P_{L}\left(d_{i}\right)}} e^{j\left(2 \pi d_{i} / \lambda-\Psi_{i}\right)}\right|^{2},
$$

where $d_{i}$ is the vector length from the $i$ th antenna element to the observation point (as in above), term $\exp \left(j 2 \pi d_{i} / \lambda\right)$ accounts for the linear phase shift due to propagation, $\Psi_{i}$ are the antenna phases and are taken as $(i-1) \Delta$ for the LPBS and as $\phi_{i}$ from (3) for the NFF, and $G_{R x}$ is the receiving antenna gain. This expression is generally valid for NF and FF, but per-antenna path losses and linear phase shifts are required in the NF due to spherical signal propagation close to the antenna array. In the FF, this equation reduces to familiar

$$
P_{R X}=\frac{P_{T x} G_{R X}}{P_{L}(r)}\left|A_{f}(\bar{r})\right|^{2},
$$

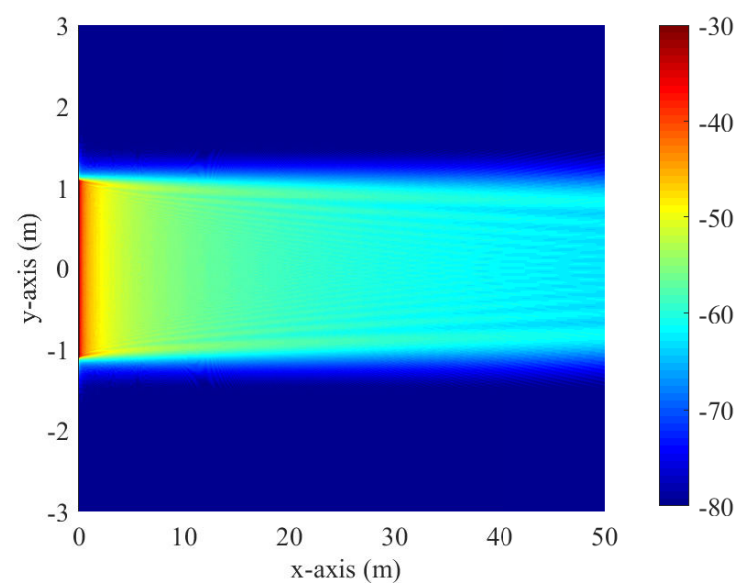

Fig. 3. Received power $(\mathrm{dBm})$ radiated by a Linear Phase Beam Steering (LPBS) array $\left(\theta_{s}=0\right)$

where $r$ is the distance to the observation point and $\bar{r}$ is the observation point about which the array factor has been calculated (given perfect alignment of the Rx antennas).

\section{SNR calculation}

In order to calculate the signal-to-noise ratio (SNR), we need to calculate the noise power. Based on the receiver noise figure $\left(R_{\mathrm{NF}}\right)$ information, the noise floor $N_{\text {floor }}$ is calculated as

$$
N_{\text {floor }}(d B)=10 \log _{10}\left(k_{B} T B\right)+R_{\mathrm{NF}},
$$

where $k_{B}$ is the Boltzmann constant, $T$ is the temperature, and $B$ is the signal bandwidth. The SNR is then calculated as

$$
\mathrm{SNR}=\frac{P_{R X}}{N_{\text {floor }}} .
$$

Based on (7), received power with LPBS and NFF approach are shown in Figs. 3 and 4, respectively, for 2048 element antenna array with element spacing $d=\lambda / 2$. The NFF is formed directly in front of the antenna 25 meters away from the array. These figures show a significant difference between two approaches in terms of power distribution. A clear maximum is formed at focus point with NFF whereas LPBS forms more uniform radiation pattern. It can be concluded that the transmitted power can be concentrated to a limited region utilizing NFF approach. For more details on the power profiles, authors in [6] provide design curves and performance data for NFF planar array, and for comparison unfocused array are taken into consideration. In [10], performance comparison of focused and unfocused antenna array is presented as well.

In addition to Figs. 3 and 4, Fig. 5 shows the received power as a function of distance for NFF and LPBS. In the NF region, the received power by NFF approach is superior to that of the LPBS, but the both methods converge to the same gain in the FF. In the NF, at user positions, $10 \mathrm{~m}, 30 \mathrm{~m}$ and $50 \mathrm{~m}$, NFF has $24 \mathrm{~dB}, 18 \mathrm{~dB}$, and $15 \mathrm{~dB}$ more received power, respectively, when compared to LPBS. This is very promising gain in the ideal case. Next, we consider breaking the ideal response with uncertainty in the user position. 


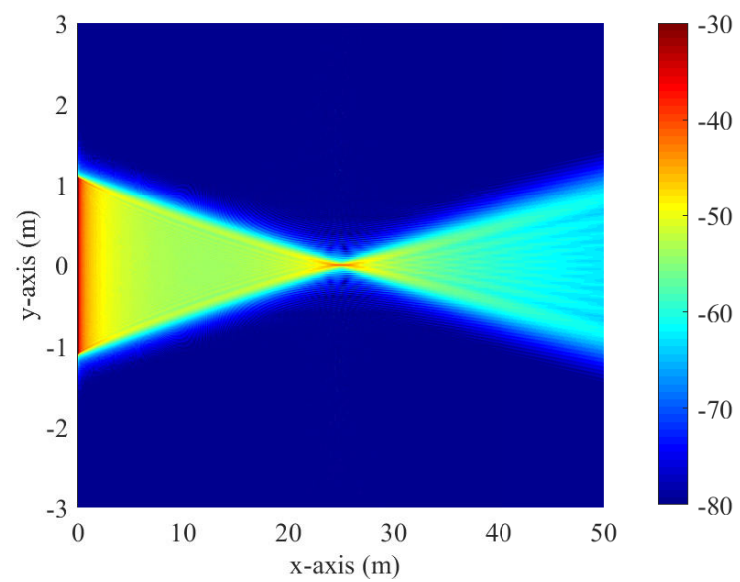

Fig. 4. Received power $(\mathrm{dBm})$ radiated by a Near Field focused (NFF) linear array (focus: $x=25 \mathrm{~m}, y=0 \mathrm{~m}$ )

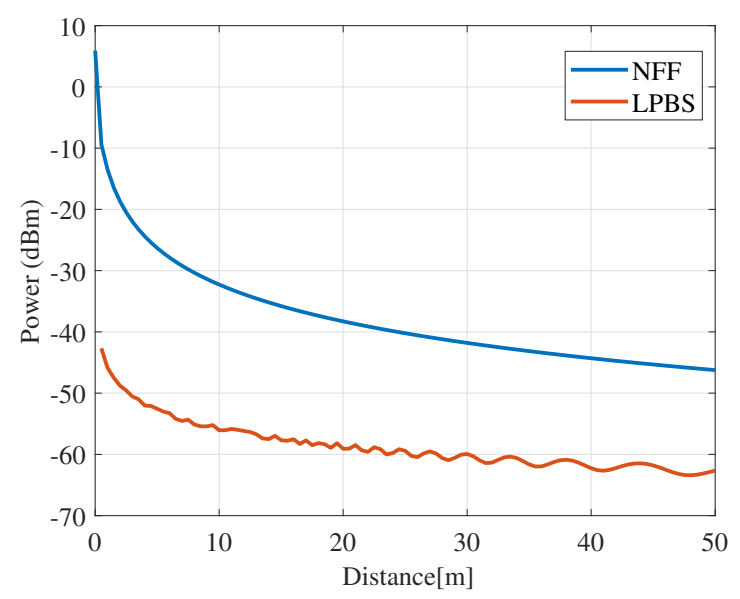

Fig. 5. Received power comparison between NFF and LPBS as a function of the user location on $x$-axis. Focus equals the user location, $\theta_{s}=0$, and $y=0 \mathrm{~m}$.

\section{E. User Position Model}

When designing a high frequency wireless system, it is crucial to think localization and user tracking. In this work, we analyze the impact of the imperfect user position information on the achievable gain for NFF and LPBS. We analyze the user position uncertainty by assuming uniform distribution for user position inside a disk of radius $r_{u}$ as shown in Fig. 1. This can be done by using disk point picking principle [15]. In the simulation model, for a certain user location, $M$ number of points are defined inside a disk over which the average received power can be calculated. Based on angle and random distance from center of the disk, $\mathrm{x}$ and $\mathrm{y}$ components for the $M$ points inside the disk can be calculated as

$$
\begin{aligned}
& X_{k}=\sqrt{r_{k}} \cos \left(\theta_{k}\right), \\
& Y_{k}=\sqrt{r_{k}} \sin \left(\theta_{k}\right),
\end{aligned}
$$

where $\theta_{k}$ and $r_{k}$ are drawn from uniform distributions; radius $r_{k}$ from zero to $r_{u}^{2}$ and angle from zero to $2 \pi$. In this work we
TABLE I

PARAMETERS USED FOR THE NUMERICAL RESULTS

\begin{tabular}{||l|l||}
\hline Parameter & Value \\
\hline \hline Center frequency $\left(f_{c}\right)$ & $140 \mathrm{GHz}$ \\
\hline Number of antennas $(N)$ & 2048 \\
\hline Transmit power $\left(P_{\mathrm{Tx}}\right)$ & 1 watt \\
\hline Wavelength $(\lambda)$ & $2.1414 \mathrm{~mm}$ \\
\hline Antenna element spacing $(d)$ & $1.2071 \mathrm{~mm}$ \\
\hline Signal bandwidth $(B)$ & $1 \mathrm{GHz}$ \\
\hline Antenna array size $(D)$ & $2.117 \mathrm{~m}$ \\
\hline Receiver noise figure $\left(R_{\mathrm{NF}}\right)$ & $5 \mathrm{~dB}$ \\
\hline Receiving antenna gain $\left(G_{\mathrm{RX}}\right)$ & $0 \mathrm{dBi}$ \\
\hline
\end{tabular}

assume uniform distribution for the radius, but in the future work we will also consider other statistics for the user position error, such as Gaussian distribution. The position $\left(r_{i k}\right)$ from each antenna array element $i$ to the $k$ th possible user position inside the disk can be calculated as

$$
r_{i k}=\sqrt{\left(X_{k}-x_{i}\right)^{2}+\left(Y_{k}-y_{i}\right)^{2}},
$$

where $x_{i}$ and $y_{i}$ give the $i$ th antenna element position and $X_{k}$ and $Y_{k}$ represent $k$ th possible user position inside disk. For the uncertainty analysis (12) can be used in (7) to implement LPBS or NFF.

\section{NUMERICAL RESULTS}

Based on (7), the power radiated by a uniform linear antenna array is calculated for a set of parameters listed on Table I. One important note to keep in mind is position of antenna array is fixed on the y-axis. In results, we focus on SNR vs. user distance, SNR vs. uncertainty, and power distribution with different user position uncertainty for conventional beam steering and NFF techniques.

\section{A. SNR vs. link distance}

First, we investigate the SNR performance comparison of the NFF and LPBS as a function of user position along parallel (y-axis) and perpendicular ( $\mathrm{x}$-axis) paths with respect to the array position as shown in Figs. 6 and 7. In these figures, blue line represents NFF while red line represents LPBS. SNR performance as a function of uncertainty is shown. Three different uncertainties are considered, $0,10 \mathrm{~cm}$, and $50 \mathrm{~cm}$. As, uncertainty increases the received power decreases.

As expected, SNR performance with zero uncertainty is better as compared to increased uncertainty in any case. Fig.7 shows achieved SNR are substantially degraded with increased uncertainty when NFF is used. In addition, as uncertainty increases the SNR plot outspread due to averaging of power received by $M$ number of possible user positions inside circle. In fact with NFF along $\mathrm{x}$-axis, power received by users at focus point with zero, $10 \mathrm{~cm}$ and $50 \mathrm{~cm}$ uncertainty are around 39, 25, and $22 \mathrm{~dB}$ respectively. For LPBS along $\mathrm{x}-$ axis, received power at $25 \mathrm{~m}$ distance are around $19 \mathrm{~dB}$ for zero, $10 \mathrm{~cm}$ and $50 \mathrm{~cm}$ uncertainty. Hence, similar SNR performance is noticed within three uncertainty cases, while 


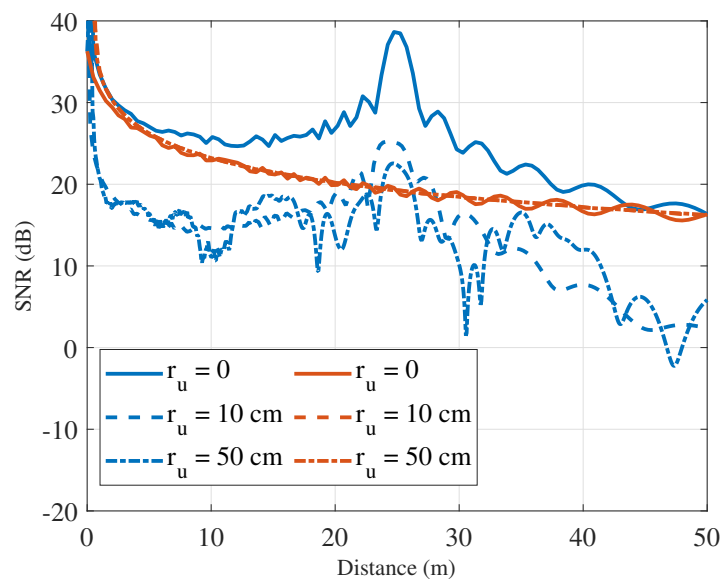

Fig. 6. Calculated SNR as a function of user location on the $\mathrm{x}$-axis for different uncertainty radii $r_{u}$ when $y=25 \mathrm{~m}$. Blue curves represent NFF (focus: $x=25 \mathrm{~m}, y=0 \mathrm{~m})$ and orange curves represent $\operatorname{LPBS}\left(\theta_{s}=0\right)$.

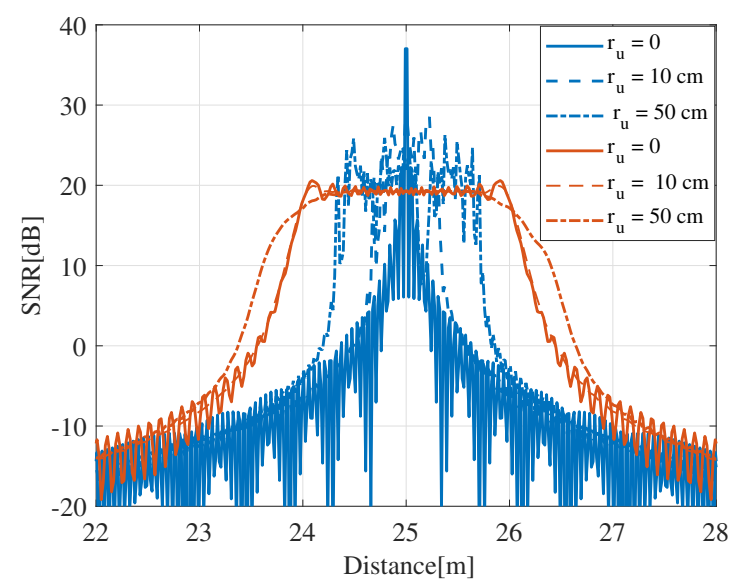

Fig. 7. Calculated SNR as a function of user location on the y-axis for different uncertainty radii $r_{u}$ when $x=25 \mathrm{~m}$. Blue curves represent NFF (focus: $x=25 \mathrm{~m}, y=0 \mathrm{~m}$ ) and orange curves represent $\operatorname{LPBS}\left(\theta_{s}=0\right)$.

greater uncertainty is vulnerable for NFF. This concludes that NFF allow improvement in achievable performance with low user uncertainty, but as uncertainty increases, LPBS performs very well when compared to NFF. In the similar way SNR performance comparison for user along y-axis is shown with same uncertainty considerations. The results herein reported denote that user uncertainty consideration is crucial when analyzing the NFF antenna array.

\section{B. SNR Vs. Uncertainty}

The simulated SNR figures for NFF and LPBS as a function of uncertainty radius $r_{u}$ is given in Fig. 8. The average SNR is given as the average over $M$ possible user positions inside the disk of radius $r_{u}$. For this figure, the NFF is focused on $(\mathrm{x}, \mathrm{y})$ position $(25 \mathrm{~m}, 0 \mathrm{~m})$. In general, if an user is at focus, for lower user uncertainty $\left(r_{u}\right)<10 \mathrm{~cm}$, NFF is better as compared to LPBS. At zero uncertainty, the NFF gives the maximum SNR, which reads $38.76 \mathrm{~dB}$. The same for LPBS is $19.56 \mathrm{~dB}$. As the

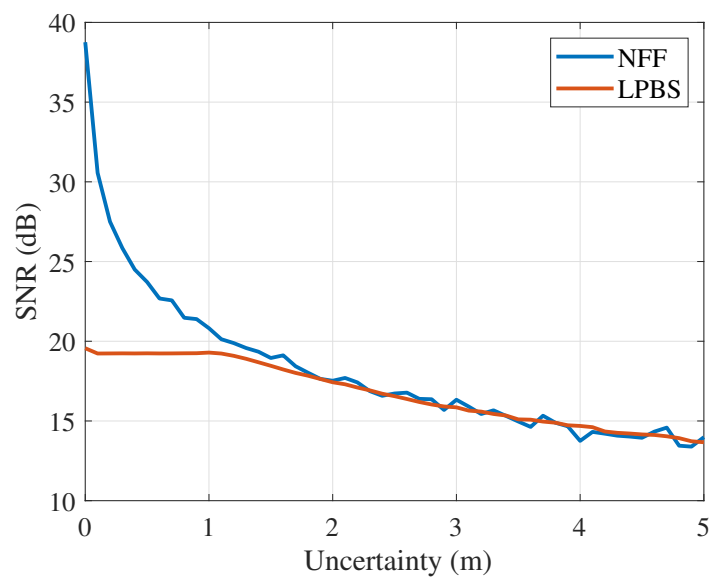

Fig. 8. SNR as a function of uncertainty $r_{u}$ for a single user. Focus equals the observation point $(x=25 \mathrm{~m}, y=0 \mathrm{~m})$

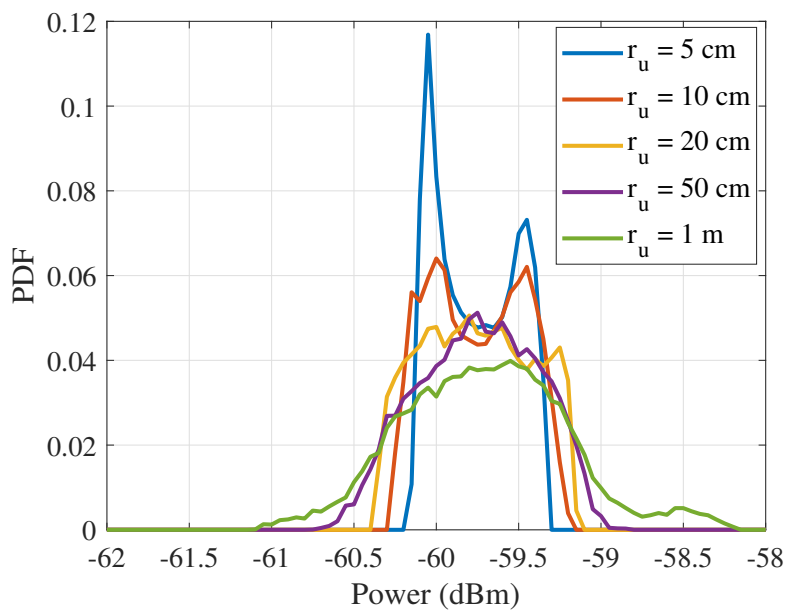

Fig. 9. LPBS power distributions for different $r_{u}$.

uncertainty increases, the SNR performance is decreasing for the both NFF and LPBS. The SNR level of LPBS antenna array gradually decreases as uncertainty increases, whereas NFF experience a sharp SNR degradation up to about $1 \mathrm{~m}$ of uncertainty, then decreases gradually. At $5 \mathrm{~m}$ uncertainty the power received by user is $24 \mathrm{~dB}$ less in NFF and $6 \mathrm{~dB}$ less in LPBS as compared to user at uncertainty $0 \mathrm{~m}$. As compared to LPBS, NFF antenna array has diminishing gain over the LPBS as uncertainty increases. Power distributions for various uncertainties are further studied below.

\section{Power Distribution with User Position Uncertainty}

Figs. 9 and 10 show the probability density functions (PDFs) of the received power for a user positioned at focus point (25 $\mathrm{m}, 0 \mathrm{~m})$. Specifically, we consider position uncertainty radii $5 \mathrm{~cm}, 10 \mathrm{~cm}, 20 \mathrm{~cm}, 50 \mathrm{~cm}$, and 1 meter. The distributions are calculated over 50,000 data points. As expected, the NFF outperforms LPBS when there is no uncertainty in the user position. Power distribution of LPBS is spread across relatively 


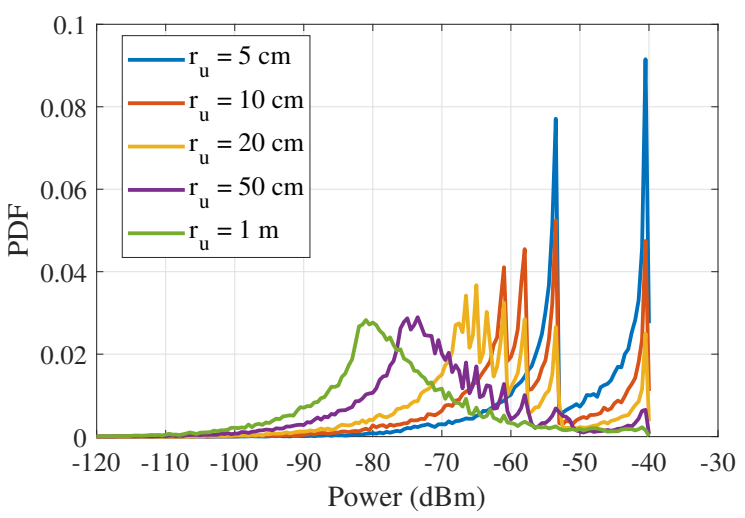

Fig. 10. NFF power distributions for different $r_{u}$.

close set of received powers. Thus, the variance of the received power is small. As compared to LPBS, NFF power distribution is more spread out. Notice that uncertainty impacts heavily on the performance of NFF antenna array, Here uniform distribution of user position uncertainties is considered, but in many real scenarios user position error is not uniformly distributed. The statistics of the user position are extremely important as NFF suffers from increased losses if there is uncertainty in the exact user position. So, this suggest more research towards uncertainty studies in future work. Indeed, the greater the uncertainty the lesser will be the gain. In the ideal, as shown in Fig. 5, NFF performs excellent when the system is ideal, but with uncertainties, traditional beamforming may be an appealing option. Therefore, the choice of the beam steering depends on application and amount and accuracy of the information we have for beamforming.

\section{Conclusions}

We analysed the impact of the NF user position uncertainty on the link performance in this paper. We compared two beamsteering methods, LPBS and NFF. The former is usually used in the FF where the relative path lengths from different antenna elements to the Rx are comparable. In the NF, these path lengths vary based on the user position. The NFF aligns the phases at the Rx and gives the maximum achievable antenna gain. Thus, the NF focusing gives superior performance in the $\mathrm{NF}$ of the antenna array. Due to focusing, the spot where the maximum gain is at, is very small. This leads to increased losses if there is uncertainty in the exact user position. This uncertainty may be caused, e.g., by user movement causing the channel estimate to become inaccurate before obtaining the next channel estimate, or by the inaccuracy of the estimate itself. Depending on the depth of the position uncertainty, whereas superior in the ideal case, the NFF can lead to worse performance than the LPBS. However, with moderate uncertainty, the NFF still gives very good performance. The future work continues from the findings shown here into more complex antenna models and more complex position uncertainty models with user mobility. Those give more ground on analysing the need for channel estimation and frequency of the estimation with different levels of mobility in order to maximize the communications performance above $100 \mathrm{GHz}$ frequencies. However, the results herein clearly show that the optimal beamforming method is dependent on the accuracy of the position information. Sometimes it may be worth to lose some gain in favor of more robust and consistent beamforming.

\section{ACKNOWLEDGMENT}

This work was supported by Horizon 2020, European Union's Framework Programme for Research and Innovation, under grant agreement no. 871464 (ARIADNE). This work has also been partly funded by the European Commission through the H2020 project Hexa-X (Grant Agreement no. 101015956) and by the Academy of Finland 6Genesis Flagship under grant no. 318927.

\section{REFERENCES}

[1] M. Latva-Aho and K. Leppänen, Eds., Key drivers and research challenges for $6 \mathrm{G}$ ubiquitous wireless intelligence, ser. $6 \mathrm{G}$ research visions. University of Oulu, Sep. 2019, no. 1.

[2] R. Hansen, "Aperture theory," in Apertures. Elsevier, 1964, pp. 1-105.

[3] A. F. Molisch, Wireless communications. John Wiley \& Sons, 2012, vol. 34 .

[4] E. Björnson and L. Sanguinetti, "Power scaling laws and near-field behaviors of massive mimo and intelligent reflecting surfaces," arXiv, 2020. [Online]. Available: https://arxiv.org/abs/2002.04960

[5] C. A. Balanis, Antenna theory: analysis and design. John wiley \& sons, 2016.

[6] A. Buffi, P. Nepa, and G. Manara, "Design criteria for near-field-focused planar arrays," IEEE Antennas and Propagation Magazine, vol. 54, no. 1, pp. 40-50, 2012

[7] R. J. Mailloux, Phased array antenna handbook. Artech house, 2017.

[8] J. Sherman, "Properties of focused apertures in the fresnel region," IRE Transactions on Antennas and Propagation, vol. 10, no. 4, pp. 399-408, 1962.

[9] H. Kamoda, T. Iwasaki, J. Tsumochi, T. Kuki, and O. Hashimoto, "60ghz electronically reconfigurable large reflectarray using single-bit phase shifters," IEEE Transactions on Antennas and Propagation, vol. 59, no. 7, pp. 2524-2531, 2011.

[10] A. Badawi, A. Sebak, and L. Shafai, "Array near field focusing," in IEEE WESCANEX 97 Communications, Power and Computing. Conference Proceedings, 1997, pp. 242-245.

[11] M. Bogosanovic and A. G. Williamson, "Microstrip antenna array with a beam focused in the near-field zone for application in noncontact microwave industrial inspection," IEEE Transactions on Instrumentation and Measurement, vol. 56, no. 6, pp. 2186-2195, 2007.

[12] V. R. Gowda, O. Yurduseven, G. Lipworth, T. Zupan, M. S. Reynolds, and D. R. Smith, "Wireless power transfer in the radiative near field," IEEE Antennas and Wireless Propagation Letters, vol. 15, pp. 18651868, 2016.

[13] S. Karimkashi and A. A. Kishk, "Focusing properties of fresnel zone plate lens antennas in the near-field region," IEEE Transactions on Antennas and Propagation, vol. 59, no. 5, pp. 1481-1487, 2011.

[14] S. Payami and F. Tufvesson, "Channel measurements and analysis for very large array systems at $2.6 \mathrm{ghz}$," in 2012 6th European Conference on Antennas and Propagation (EUCAP), 2012, pp. 433-437.

[15] E. W. Weisstein, "Disk point picking," [Online] Available : https://mathworld.wolfram.com/DiskPointPicking.html, 2002. 